\title{
Preparation and characterization of sodium alginate/acrylic acid composite hydrogels conjugated to silver nanoparticles as an antibiotic delivery system
}

https://doi.org/10.1515/gps-2021-0081

received June 25, 2021; accepted November 09, 2021

\begin{abstract}
Hydrogels are promising drug delivery systems attributable to their unique characteristics such as high hydrophilicity, controllability, biocompatibility, and facile production routines. The aim of this research was the preparation of sodium alginate/acrylic acid (AAc) composite hydrogels conjugated to silver nanoparticles to deliver the cephalexin as a model antibiotic compound. The reduction of silver ions into silver nanoparticles as well as the stabilization of created nanoparticles ensued simultaneously with hydrogel backbone formulation during microwave irradiation and monomer cross-linking processes. The impact of AAc and silver ion concentrations and the radiation time of microwave were then investigated on the main characteristics of hydrogels. The results indicated that the hydrogels' characteristics could be significantly predicted by studying all independent parameters through various second-order polynomial models. The multiple optimization analysis suggested that the prepared hydrogels using $7.8 \mathrm{~g}$ AAc and $1.5 \mathrm{~g}$ silver nitrate and $1 \mathrm{~min}$ microwave radiation could give the best hydrogels with the highest swelling degree, gel fraction, cephalexin absorption, and antibacterial activity. The morphology and either absorption or release kinetics of cephalexin by the optimum prepared hydrogels were also investigated. No significant differences between the experimental and predicted data confirmed the suitability of the suggested models.
\end{abstract}

\footnotetext{
* Corresponding author: Navideh Anarjan, Department of Engineering, Tabriz Branch, Islamic Azad University, Tabriz, Iran, e-mail: anarjan@gmail.com, anarjan@iau.ac.ir, tel: +98-9144027140, fax: +98-3868684

Parvaneh Mohamadinia: Department of Engineering, Tabriz Branch, Islamic Azad University, Tabriz, Iran

Hoda Jafarizadeh-Malmiri: Faculty of Chemical Engineering, Department of Food Engineering, Sahand University of Technology, Tabriz, Iran
}

Keywords: sodium alginate, acrylic acid, composite hydrogels, silver nanoparticles, antibiotic delivery

\section{Introduction}

Hydrogel nanocomposites are one type of biomaterials that have newly attracted a lot of consideration for their applications in environmental, medical, and pharmaceutical areas. They have been investigated for various biological applications including drug delivery, tissue engineering, antimicrobial agents, and thermal therapy [1-3]. Hydrogel nanocomposites are obtained by incorporating different types of nanoparticles, such as metallic, clay, and ceramics, into a hydrogel structure, which can offer various characteristics and capabilities to hydrogel systems [4]. The particulate material can be merged into hydrogels in different ways such as addition into monomer solution and followed by polymerization or direct mixing into hydrogel matrix after polymerization [5]. It should be noted that the polymerization process in hydrogel formation can be occurred either chemically using cross-linking agents or physically using ionizing irradiations [1].

Various either synthetic or natural polymers or monomers can give single or composite hydrogels with improved release control capabilities. For example, sodium alginate, an anionic macromolecule, that can be elicited from marine algae or produced by bacteria, is plentiful, renewable, nontoxic, water-soluble, and biocompatible polymer, with great gelation capacity. It can be readily modified by other monomers or polymers to give the most desired hydrogels for certain applications [2]. Acrylate-based hydrogels are another group of the most talented systems in the delivery of pharmaceutical constituents, such as antibacterial in wound wearing, bone cement, contact lenses, and formulations. However, due to some deficiency of their physical and biological properties, especially their hydrated state at 
the body temperature, such as antifouling activity, rigidity, and water diffusion, novel acrylic-based hydrogels with composite structures have been developed to improve their characteristics and give them superior applicability in biomedical and bioengineering uses. For example, poly (acrylic acid [AAc]) and alginate composite were produced in interpenetrating polymer network form and incorporated with silica nanoparticles, or graphene nanosheets were integrated into poly(AAc)/gelatin composite hydrogels, to produce metrics with the most desired physical and biological characteristics [6].

Although antibiotic compounds have been used to stop bacterial infections, most of them are not effective against multidrug-resistant pathogenic strains. Therefore, inorganic antibacterial agents such as silver with great activities against all wild and multidrug-resistant pathogens were proposed as a new class of antibacterial agents. The more stable and long-lasting antimicrobial activities of silver nanoparticles as compared to silver ions and microstructured silver have been confirmed in most of the previous researches [7]. Consequently, the size reduction of silver particles into nanometric scales or renovation of silver ions to silver nanoparticles can improve their antibacterial activities, considerably. It was hypothesized that the silver ions can be reduced into silver nanoparticles and also stabilized during the hydrogel formation process through the polymerization of monomers [8].

Previous researches have confirmed the synergistic antibacterial effect of silver with other antibiotic compounds [8]. Thus, due to the great ability of hydrogels in either absorption or release of bioactive compounds, it was anticipated that the composite hydrogels conjugated to silver nanoparticles could effectively absorb various antibiotics such as cephalexin and so offer efficient, stable, and strong bactericidal actions in microbial infection therapies.

Therefore, in this research, the sodium alginate/AAc composite hydrogels conjugated to silver nanoparticles were developed to absorb and encapsulate cephalexin and provide the synergistic effect in antibacterial activities of silver and organic antibiotic compounds. The effects of main formulation and process parameters, namely, AAc and silver ion (silver nitrate) concentrations, as well as the microwave irradiation time, were investigated on characteristics of produced composite hydrogels. The various polynomial models were suggested to predict the hydrogels' characteristics by selected independent parameters. Finally, the process parameters were optimized, aimed to develop the best product with the most desired properties such as the greatest swelling degree, gel fraction, drug load, and the least drug release. The morphology and either absorption or release kinetics of the optimum prepared hydrogels were studied, as well.

The synthesis of sodium alginate/AAc composite hydrogels through physical and chemical techniques, simultaneously, and using it to produce silver nanoparticles and the adsorption of a chemical antibiotic in the obtained delivery system are among the innovations of this research.

\section{Materials and methods}

\subsection{Materials}

All AAc, sodium alginate, calcium chloride, and silver nitrate were obtained from Merck (Gernsheim, Germany). All solvents and deionized water were also acquired from Dr Mojallali's company (Tehran, Iran). Cephalexin was donated from Daana Pharmaceutical Company (Tabriz, Iran).

\subsection{Methods}

The hydrogels were produced by simultaneously chemically cross-linking of sodium alginate by calcium chloride and physically polymerizing and cross-linking of AAC monomers by microwave irradiation.

Silver nitrate was dissolved in $5 \mathrm{~mL}$ deionized water and slowly added to a $50 \mathrm{~mL}$ aqueous solution of AAc. Various quantities of silver nitrate and AAc were used in the preparation of each sample according to the experimental design (Table 1). The mixture was extra stirred magnetically for $30 \mathrm{~min}$ before subjecting to microwave irradiation (LG NeoChef ${ }^{\mathrm{TM}}$ countertop microwaves with Smart Inverter, LG Corporation, Seoul, South Korea). The duration of microwave irradiation was done according to the design of the experiment (Table 1), with the difference that the time of irradiation was intermittent, and between every $30 \mathrm{~s}, 1 \mathrm{~min}$ of rest was given to prevent the overheating of the samples. After the microwave irradiation process, the sample was dropwise added into an aqueous solution of sodium alginate ( $3.5 \mathrm{~g}$ dissolved sodium alginate in $100 \mathrm{~mL}$ deionized water) and mixed completely under a magnetic stirrer for an extra $1 \mathrm{~h}$. At last, the calcium chloride solution (11 $\mathrm{g}$ dissolved calcium chloride in $50 \mathrm{~mL}$ deionized water) was added into the system at the rate of $5 \mathrm{~mL} \cdot \mathrm{min}^{-1}$ under a magnetic stirrer. The mixing of samples was continued for an extra $30 \mathrm{~min}$, and then they were dried in an electrical oven at $5^{\circ} \mathrm{C}$ for 3 days. The synthesis procedures were shown in Scheme 1, schematically. 
Table 1: Design of experiment and independent and response variables in the preparation of alginate/AAc composite hydrogels conjugated to silver nanoparticles

\begin{tabular}{|c|c|c|c|c|c|c|c|}
\hline Sample & $\begin{array}{l}\text { Acrylic } \\
\text { acid (g) }\end{array}$ & $\begin{array}{l}\text { Silver } \\
\text { nitrate (g) }\end{array}$ & $\begin{array}{l}\text { Microwave } \\
\text { time (min) }\end{array}$ & $\begin{array}{l}\text { Swelling } \\
\text { ratio (\%) }\end{array}$ & $\begin{array}{l}\text { Gel } \\
\text { fraction }\end{array}$ & $\begin{array}{l}\text { Cephalexin } \\
\text { load }(\%)\end{array}$ & $\begin{array}{l}\text { Clear } \\
\text { zone }(\mathrm{mm})\end{array}$ \\
\hline 1 & 7.97 & 1 & 4.1 & 148.4 & 0.070 & 92.00 & 21 \\
\hline 2 & 0 & 0.5 & 3 & 108.7 & 0.310 & 59.25 & 13 \\
\hline 3 & 0.05 & 0.5 & 3 & 105.6 & 0.230 & 57.05 & 13 \\
\hline 4 & 7.97 & 1 & 1.8 & 506.7 & 0.520 & 98.75 & 21 \\
\hline 5 & 0.05 & 1 & 3 & 124.4 & 0.440 & 64.50 & 16 \\
\hline 6 & 2.02 & 0.5 & 4.1 & 257.7 & 0.340 & 43.75 & 16 \\
\hline 7 & 2.02 & 1 & 1.8 & 392.2 & 0.690 & 58.75 & 17 \\
\hline 8 & 0.1 & 0.3 & 3 & 10.0 & 0.236 & 57.75 & 12 \\
\hline 9 & 0.05 & 1.5 & 1.8 & 50.0 & 0.910 & 53.00 & 21 \\
\hline 10 & 7.97 & 0.3 & 1.8 & 107.0 & 0.200 & 55.00 & 19 \\
\hline 11 & 2.02 & 0.5 & 1.8 & 203.7 & 0.340 & 46.00 & 20 \\
\hline 12 & 2.02 & 1 & 4.1 & 70.0 & 0.230 & 58.50 & 19 \\
\hline 13 & 0.05 & 0.5 & 1 & 135.3 & 0.280 & 56.75 & 16 \\
\hline 14 & 0.05 & 0.5 & 3 & 105.7 & 0.230 & 60.25 & 12 \\
\hline 15 & 0.05 & 0.7 & 3 & 170.5 & 0.290 & 66.00 & 14 \\
\hline 16 & 0.05 & 0.5 & 5 & 165.1 & 0.260 & 51.00 & 10 \\
\hline 17 & 0.05 & 1 & 3 & 30.0 & 0.490 & 72.75 & 15 \\
\hline 18 & 7.97 & 0.3 & 4.1 & 261.3 & 0.250 & 51.25 & 17 \\
\hline 19 & 0.05 & 0.5 & 3 & 106.0 & 0.210 & 61.50 & 12 \\
\hline 20 & 0.05 & 0.5 & 3 & 105.4 & 0.240 & 59.25 & 13 \\
\hline
\end{tabular}

\subsection{Experimental design}

The response surface method with the central composite design was used to analyze variance using analysis of variance (ANOVA), multiple regression analysis, and multigoal optimization to get the most desired hydrogels with the maximum swelling degree, gel fraction, cephalexin load, and antibacterial activities (response variables). The significant effects of independent variables, either in linear, quadratic, or interaction forms, at a 95\% confidence interval ( $p$-value $<0.05)$ were also determined based on their obtained $p$-value and $F$-ratio through ANOVA. By multiple regression analysis, the polynomial models were provided for response variables to predict their changes by selected independent variables. The terms with the smaller $p$-values (less than 0.05 ) and greater $F$-ratio are considered as the more significant effect. The selected independent variables were the AAc and silver nitrate (silver ions) contents and the radiation time of the microwave. Consequently, 20 composite hydrogels were prepared at different five levels of AAc and silver ion concentrations and microwave irradiation time. The sample preparation order was randomized, and the center point was repeated various times to prevent systematic errors and confirm the repeatability of samples. Besides, the response variables' contour plots were also provided to visualize their changes by selected formulation and process parameters [9].

\subsection{The performed analysis on all samples}

\subsubsection{Swelling ratio}

About $1 \mathrm{~g}$ of each dried sample was immersed in deionized water for $8 \mathrm{~h}$. The remained solution was completely removed from swollen hydrogels by filter paper, and then the swelling ratio of hydrogels was calculated using Eq. 1:

$$
\text { Swelling ratio }(\mathrm{w} / \mathrm{w})=\frac{w_{2}-w_{1}}{w_{1}} \times 100=(\mathrm{g} / \mathrm{g})
$$

where $w_{1}$ and $w_{2}$ are initial dry hydrogel and swelled hydrogel weights, respectively [10].

\subsubsection{Gel fraction}

The swelled hydrogels were placed in aluminum plates and put into the oven for $10 \mathrm{~h}$ at $55^{\circ} \mathrm{C}$ until their complete dehydration. Then, they were carefully weighted. Gel fraction is calculated from Eq. 2:

$$
\text { Gel fraction }=\frac{w_{3}}{w_{2}} \times 100
$$

where $w_{2}$ and $w_{3}$ are the swelled hydrogels and dried weights, respectively [10]. 


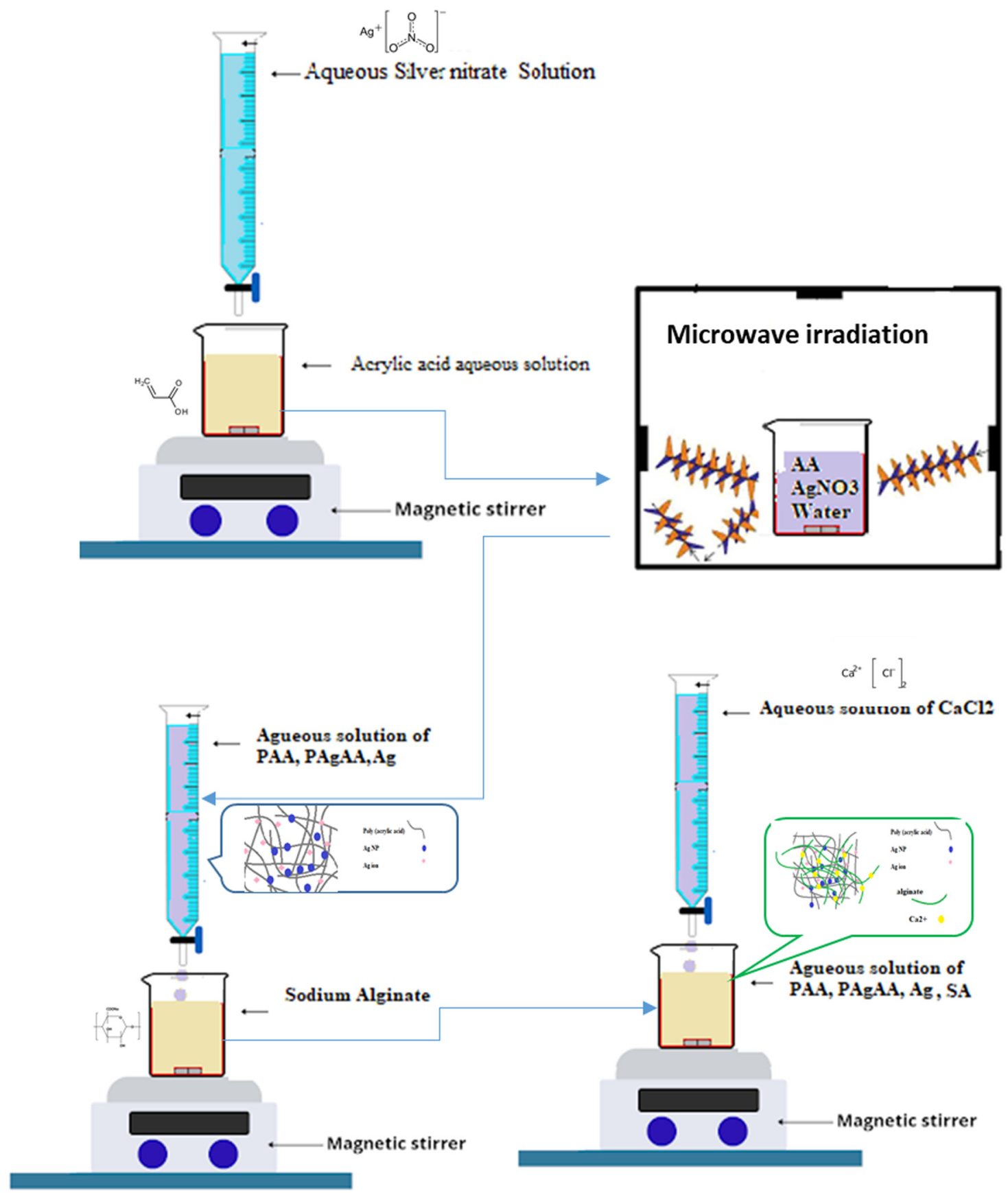

Scheme 1: The schematic for the synthesis of the sodium alginate/AAc composite hydrogels conjugated to silver nanoparticles.

\subsubsection{Cephalexin load}

About $1 \mathrm{~g}$ of each dried hydrogel was dipped into a $15 \mathrm{~mL}$ $\mathrm{NaOH}$ solution $(0.1 \mathrm{~N})$ containing $0.5 \mathrm{~g}$ cephalexin for $24 \mathrm{~h}$ to complete the absorption process of cephalexin. The cephalexin solution was then centrifuged and neutralized, and the light absorption intensity of the supernatant was measured using a UV-Vis spectrophotometer (DR5000, Hack, Canada) at $\lambda=320 \mathrm{~nm}$ to find the remained (unabsorbed) cephalexin of solution. The unadsorbed cephalexin content in the solution was determined based on the previously provided standard curve $\left(c_{\mathrm{r}}\right)$. The cephalexin load of samples was then calculated by subtraction of obtained cephalexin content $\left(c_{\mathrm{r}}\right)$ from its initial content (0.5), according to Eq. 3. The $\mathrm{NaOH}$ aqueous solution $(0.1 \mathrm{~N})$ was considered a blank sample.

$$
\text { Cephalexin load }(\%)=\frac{0.5-c_{\mathrm{r}}}{0.5} \times 100
$$




\subsubsection{Antibacterial test against Staphylococcus aureus}

The antibacterial activity of hydrogels was also tested against $S$. aureus (aerobic gram-positive bacteria) using the agar well diffusion technique. Various $8 \mathrm{~mm}$ diameter wells have been introduced aseptically to inoculated nutrient agar with $S$. aureus in a certain concentration (0.5 McFarland). Then, $10 \mathrm{mg}$ of each hydrogel was dissolved in $10 \mu \mathrm{L}$ deionized water and poured into the wells. The agar plates were incubated at $37^{\circ} \mathrm{C}$ for $48 \mathrm{~h}$. The cephalexin diffuses into the agar medium and hinders the growth of bacteria around the wells and can be observed as a clear zone. The clear zone diameter is a good indicator of the antibacterial activity of hydrogels as a bigger clear zone corresponds to the greater antimicrobial activity. The experiments were performed in triplicate, and the mean clear zone radial was reported as the antimicrobial activity of samples. The antibacterial activities of hydrogels with no incorporated cephalexin were evaluated as a blank sample [11].

\subsection{The performed analysis on the optimum formulated hydrogel}

\subsubsection{Cephalexin absorption kinetics}

To determine the cephalexin absorption kinetics, $0.5 \mathrm{~g}$ of optimum hydrogel was added into a $50 \mathrm{~mL} \mathrm{NaOH}$ solution $(0.1 \mathrm{~N})$ containing $0.25 \mathrm{~g} \cdot \mathrm{L}^{-1}$ cephalexin. The light absorption intensity of the solution was measured in $4 \mathrm{~min}$ intervals for $5 \mathrm{~h}$, at $\lambda=320 \mathrm{~nm}$ using a UV-Vis spectrophotometer (DR5000, Hack, Canada). Based on the stablished standard curve, it is possible to calculate of the cephalexin contents in the solution as manifested in the solution absorption intensity. The loaded cephalexin by hydrogels each time was coded as $C_{\mathrm{a}}$ and calculated as $C_{\mathrm{a}}=I_{\text {initial }}-C_{t}$ 'where $I_{\text {initial }}$ is $0.25 \mathrm{~g} \cdot \mathrm{L}^{-1}$, and the $C_{t}$ is the measured concentration of cephalexin in solution at time $t$.

\subsubsection{Cephalexin release kinetics}

For the investigation of cephalexin release, $0.5 \mathrm{~g}$ of optimum hydrogel was dipped into $15 \mathrm{~mL} \mathrm{NaOH}$ solution $(0.1 \mathrm{~N})$ containing $0.5 \mathrm{~g}$ cephalexin for $24 \mathrm{~h}$. Then, the loaded hydrogel was placed in an aqueous solution with a $\mathrm{pH}$ of 7.4 and room temperature $\left(25 \pm 4^{\circ} \mathrm{C}\right)$. The light absorption intensities of this solution were measured in $4 \mathrm{~min}$ intervals for $5 \mathrm{~h}$, at $\lambda=320 \mathrm{~nm}$ using a UV-Vis spectrophotometer (DR5000,
Hack, Canada). The light absorption intensity of the solution was converted to cephalexin content, using a standard curve, and marked as released cephalexin from hydrogel into the solution at time $t$.

\subsubsection{Fourier-transform infrared (FT-IR) spectroscopy}

FT-IR spectroscopy was used to determine the functional groups in the optimum product chemical structure. The dried hydrogel was ground, and the spectra were achieved against potassium bromide using JUSCO spectrophotometer (JUSCO 4100, Japan) at $\lambda$ ranged from 4,000 to $400 \mathrm{~cm}^{-1}$. The analyses were performed in transmittance mode.

\subsubsection{UV-Vis absorption spectra}

To confirm the formation of silver nanoparticles into sodium alginate/AAc composite hydrogel networks, the UV-Vis absorbance spectra of the optimum product were recorded. The optimum product was first dispersed in deionized water, and then its absorption spectra were recorded at $25^{\circ} \mathrm{C}$ using UV-Vis spectrophotometer (DR5000, Hack, Canada), in wavelength from 250 to $800 \mathrm{~nm}$.

\subsubsection{Scanning electron microscope (SEM)}

The morphology of optimum hydrogel was also evaluated using field-emission scanning electron microscopy (FE-SEM, VEGA//TESCAN, Czech Republic). About $0.1 \mathrm{~g}$ of optimum hydrogel was placed in a copper grid and coated with gold to increase the conductivity of samples. The micrographs were then provided.

\section{Results and discussion}

\subsection{Swelling ratio and gel fraction}

The sodium alginate/AAc composite hydrogels conjugated to silver nanoparticles were successfully synthesized through a simultaneous chemically ion cross-linking and physically microwave polymerization of sodium alginate and AAc, respectively. However, based on used AAc and silver ion contents and applied microwave time, their characteristics differed considerably. For instance, the swelling ratio of the obtained hydrogels varied from $10 \%$ to $506.7 \%$, and their gel fraction lay between 0.07 and 0.91 . All gained hydrogels 
Table 2: ANOVA results and $p$-values and $F$-ratio of linear, quadratic, and binary interaction terms in suggested models for prepared sodium alginate/AAc composite hydrogels conjugated to silver nanoparticles

\begin{tabular}{lllllllllllllll}
\hline Characteristics & $\begin{array}{l}\text { Linear } \\
\text { effects }\end{array}$ & $\boldsymbol{X}_{\mathbf{1}}$ & $\boldsymbol{X}_{\mathbf{2}}$ & $\boldsymbol{X}_{\mathbf{3}}$ & $\begin{array}{l}\text { Quadratic } \\
\text { effects }\end{array}$ & $\boldsymbol{X}_{\mathbf{1 1}}$ & $\boldsymbol{X}_{\mathbf{2 2}}$ & $\boldsymbol{X}_{\mathbf{3 3}}$ & $\begin{array}{l}\text { Interaction } \\
\text { effects }\end{array}$ & $\boldsymbol{X}_{\mathbf{1 2}}$ & $\boldsymbol{X}_{\mathbf{1 3}}$ & $\boldsymbol{X}_{\mathbf{2 3}}$ \\
\hline Swelling & $p$-value & 0.000 & 0.000 & 0.000 & 0.000 & 0.000 & 0.000 & 0.000 & 0.008 & 0.000 & 0.234 & 0.458 & 0.000 \\
& F-ratio & 302.91 & 320.91 & 166.80 & 534.15 & 163.31 & 46.40 & 321.43 & 11.11 & 199.71 & 1.61 & 0.60 & 565.49 \\
Gel fraction & $p$-value & 0.000 & 0.000 & 0.000 & 0.000 & 0.025 & 0.007 & 0.708 & 0.756 & 0.000 & 0.021 & 0.135 & 0.000 \\
& -ratio & 4106 & 10846 & 76.6 & 31.63 & 4.8 & 11.65 & 0.15 & 0.10 & 34.68 & 7.5 & 2.64 & 90.07 \\
Cephalexin & $p$-value & 0.000 & 0.000 & 0.000 & 0.355 & 0.000 & 0.000 & 0.013 & 0.017 & 0.001 & 0.000 & 0.373 & 0.507 \\
load & F-ratio & 39.23 & 107.20 & 33.69 & 0.94 & 26.59 & 60.42 & 9.03 & 8.27 & 12.11 & 35.81 & 0.87 & 0.47 \\
Clear zone & $p$-value & 0.000 & 0.000 & 0.001 & 0.671 & 0.24 & 0.005 & 0.078 & 0.681 & 0.167 & 0.800 & 0.291 & 0.071 \\
& $F$-ratio & 15.31 & 35.85 & 20.23 & 0.19 & 4.93 & 12.60 & 3.84 & 0.18 & 2.07 & 0.07 & 1.24 & 4.08 \\
\hline
\end{tabular}

exhibited noticeable bactericidal activities due to the presence of silver in their matrice and were able to encapsulate and load various compounds such as cephalexin from $36.89 \%$ to $92.58 \%$. The characteristics of all samples prepared in different conditions were shown in Table 1, and the ANOVA results were summarized in Table 2.

The ability to be swollen in thermodynamically compatible media is the most favorable characteristic of hydrogels. The water molecules penetrate the polymeric network of hydrogels and the rubbery phase of hydrogels, which is first be expanded and filled by penetrated molecules of solvent and detached from glassy segments. In contradiction of the favorable osmotic force, the opposite elasticity force occurs that stopovers the extending and deformation of the hydrogel networks. The equilibrium in swelling will occur as the elasticity and osmotic forces balance [12].

The ANOVA results for the swelling ratio of prepared hydrogels indicated that all selected process parameters affected the swelling ratio of hydrogels significantly, especially in linear form. The interactions of AAc with silver nitrate and microwave time were insignificant terms on this characteristic. Thus, these two terms were removed from the initial model, and the final reduced model was offered as Eq. 4:

Swelling ratio $(\%)$

$$
\begin{aligned}
= & -742.5+63.40 X_{1}+2255.1 X_{2}+108.4 X_{3} \\
& -4.879 X_{1}^{2}-876.8 X_{2}^{2}+9.89 X_{3}^{2}-314.9 X_{2} \cdot X_{3}
\end{aligned}
$$

The coefficient of determination $\left(R^{2}\right)$ for this model was $97.26 \%$. Thus, it can be concluded that this model can successfully predict about $97 \%$ of swelling ratio changes of hydrogels. Moreover, the insignificant $p$-value of lack of fit $(p$-value $=0.824)$ can confirm the suitability of this model in predicting the swelling ratio of sodium alginate/AAc composite hydrogels. By comparing the $F$-ratio of significant terms, it could be also concluded that the interaction effect of silver nitrate and microwave time had the greatest effects on this characteristic (Table 2).

The main effect plot of independent variables on the swelling ratio of hydrogels was shown in Figure 1a. Since the interaction of silver nitrate with microwave time was
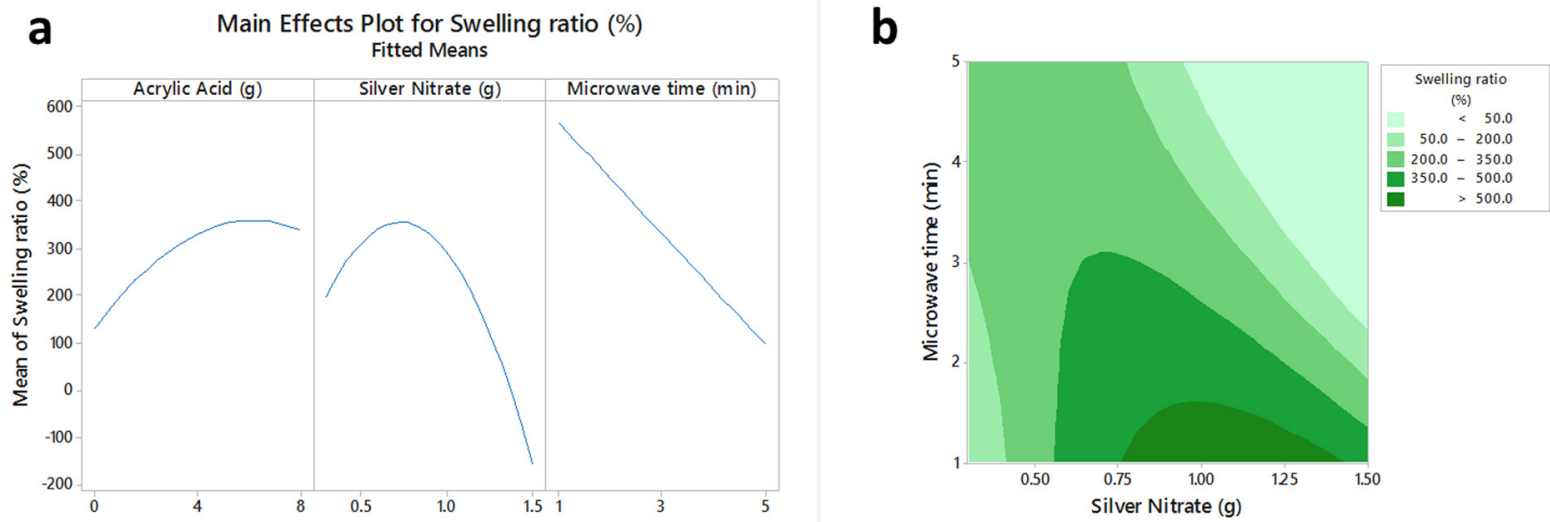

Figure 1: The main effects plot (a) and the contour plot (b) of acrylate acid, silver nitrate and microwave time on swelling ratio of sodium alginate/acrylic acid composite hydrogels conjugated to silver nanoparticles. 
only the significant term on swelling ratio, just the contour plot of the variation of this response by silver nitrate and microwave time at the certain level of AAc $(3.985 \mathrm{~g})$ was visualized as Figure 1b. As can be seen in Figure 1a, using greater AAc contents in the formulation of hydrogels could yield a product with a larger swelling ratio. An optimum content was obtained for silver nitrate leading to the hydrogels with the highest swelling ratio. Thus, the swelling ratio of hydrogels increased by rising the silver ions up to certain levels, and greater silver ions decreased this characteristic, considerably. The swelling ratio of hydrogels also decreased by increasing microwave time. However, the effect of microwave time on swelling ratio was different at various levels of silver ion, as simultaneous increases or decreases of microwave time and silver ion content resulted in the production of hydrogels with less swelling ability.

Generally, the microwave exposure of hydrogels for a long time leads to a considerable increase in the polymerization degree of the AAc monomers. Therefore, the hydrogel network becomes more cohesive and stronger, and water penetration into the network will be limited $[13,14]$. Furthermore, the previous researches also confirmed that the presence of silver ions has a positive impact on the construction of stable AAc hydrogel matrices by accelerating the polymerization of AAc. Thus, the gained stable hydrogel matrices would have greater water absorption and swelling ability. However, at high silver ion contents, various cross-linking can be occurred between silver ions and carboxylic groups of poly(AAc), leading to form $\mathrm{O}-\mathrm{Ag}-\mathrm{O}$ bonds, in which, with the formation of these bonds, the free hydroxyl groups on poly(AAc) would be reduced. Consequently, the chance for hydrogen bonding between hydrogels carboxylic groups and water decreased, resulting in an extensive decline in water absorption of hydrogels $[6,15]$. The increase of swelling ratio by AAc content would also be related to the formation of more impregnated poly(AAc) matrices into sodium alginate networks. Therefore, the amount of empty media of hydrogel networks, which can be filled by water, increased $[6,16]$. Moreover, the swelling of hydrogels lasts until the equilibrium state, where the Gibbs free energy of hydrogel is minimized. According to the theory of Flory-Rehner, the Gibbs free energy will be minimized if the osmotic and elasticity forces become equal. An increase in hydrogel cross-linking density causes the formation of smaller chains. The shorter chains have less elasticity force as compared to longer ones. Thus, the equilibrium between elasticity and osmotic forces occurs at less swelling ratios [16].

The ANOVA results of gel fraction of prepared hydrogels showed that all considered process parameters affected the gel fraction of hydrogels pointedly, particularly in linear form. The quadratic effects of both silver nitrate and microwave time, and the interaction effect of AAc and microwave time, were insignificant terms on gel fraction changes of samples. Thus, these three terms were removed from the initial model, and the final reduced model for estimating the hydrogels' gel fractions was shown as Eq. 5, with the $R^{2}$ equal to $96.90 \%$.

$$
\begin{aligned}
\text { Gel fraction }= & -0.0627+0.09253 X_{1}+0.3005 X_{2} \\
& +0.07332 X_{3}-0.001391 X_{1}^{2} \\
& -0.00483 X_{1} X_{2}-0.06396 X_{2} X_{3}
\end{aligned}
$$

Moreover, the insignificant $p$-value of lack of fit ( $p$-value $=0.28)$ can confirm the correctness of the model in predicting the gel fraction of produced composite hydrogels. By comparing the $F$-ratio of significant terms, it can also be concluded that the linear effect of microwave time had the highest influence on this characteristic (Table 2).

The main effect plot of independent variables on the swelling ratio of hydrogels was shown in Figure 2a. The contour plots of gel fraction changes by both silver nitratemicrowave time (fixed middle level of AAc, Figure $2 b$ ) and AAc-silver nitrate (fixed middle level of microwave time, Figure 2c) were shown due to the significant effects of AAc-silver nitrate and silver nitrate-microwave time interactions. According to Figure 2a, increasing either AAc or silver nitrate and microwave time raised the gel fraction of obtained hydrogels. Moreover, simultaneous increase or decrease of AAc and silver nitrate, at constant microwave exposure time, or simultaneous increase or decrease of silver nitrate and microwave time, at a fixed level of AAc, also caused a decrease in gel fraction of samples (Figure $2 \mathrm{~b}$ and $\mathrm{c}$ ).

Increasing the AAc content led to the construction of the denser and more robust hydrogel matrices, which are insoluble in water. Furthermore, increasing the silver nitrate augmented the $\mathrm{O}-\mathrm{Ag}-\mathrm{O}$ cross-linking between the monomers and thus made them insoluble in water. The exposure of hydrogels to microwaves for an extended time also increased the polymerization degree of the AAc monomers and produced stronger water-insoluble matrices [17].

\subsection{Cephalexin load}

All synthesized hydrogels could efficiently adsorb cephalexin, ranging from $43.75 \%$ to $98.75 \%$. The ANOVA results for cephalexin load of samples indicated that the linear effects of AAc and silver nitrate concentrations, the quadratic effects of all studied independent variables, and the 

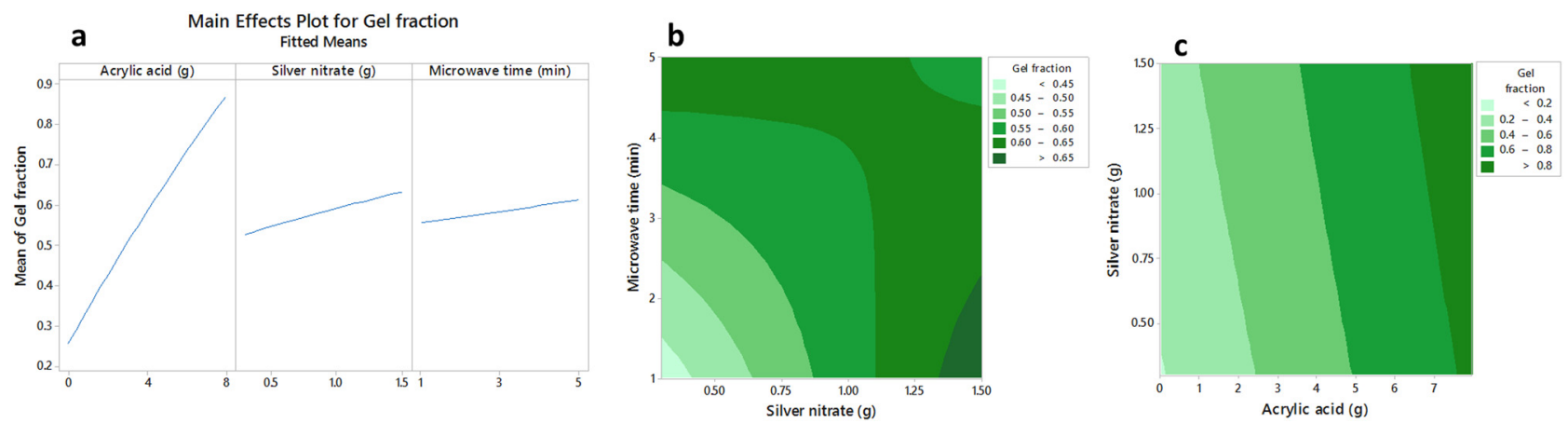

Figure 2: The main effects plot ( $a$ ) and the contour plots ( $b$ and c) of acrylate acid, silver nitrate and microwave time on gel fraction of sodium alginate/acrylic acid composite hydrogels conjugated to silver nanoparticles.

interaction effect of AAc with silver nitrate contents were significant ( $p$-value $<0.05$ ) on this response. However, the AAc content was the most effective parameter on the drug loading of samples. The final reduced model predicting the cephalexin loading of hydrogels was found as Eq. 6 . The linear effect of microwave time was not removed from the model due to its significant quadratic effect. The $R^{2}$ for this model was $96.66 \%$.

$$
\begin{aligned}
\text { Cephalexin load }(\%)= & 24.82-11.53 X_{1}+65.8 X_{2} \\
& +9.63 X_{3}+1.278 X_{1}^{2}-35.37 X_{2}^{2} \\
& -1.836 X_{3}^{2}+5.146 X_{1} X_{2}
\end{aligned}
$$

The obtained high $R^{2}$, and insignificant $p$-value of lack of fit ( $p$-value $=0.179$ ), could confirm the precision of the model in calculating the cephalexin load of produced composite hydrogels. By comparing the $F$-ratio of significant terms, it can also be concluded that the linear effect of AAc had the utmost impact on this characteristic (Table 3).

Table 3: The kinetics of cephalexin adsorption by optimum composite hydrogels (the $R^{2}$ and main coefficients for correlated general absorption models)

\begin{tabular}{lll}
\hline Common kinetic models & & Cephalexin adsorption \\
\hline Rate $=K$ & Equation & $C=0.6612 t+1.4054$ \\
Linear & $R^{2}$ & 0.9739 \\
Rate $=K C$ & Equation & $\ln C=0.2615 t+0.4047$ \\
First order & $R^{2}$ & 0.9147 \\
Rate $=K C^{2}$ & Equation & $1 / C=-0.491 t+2.3955$ \\
Second order & $R^{2}$ & 0.6630 \\
Rate $=\frac{K C}{K C+V m}$ & Equation & $1 / C=y=0.2537(1 / t)+$ \\
Michaelis-Menten & $R^{2}$ & 0.9576 \\
Rate $=K C^{n}$ & Equation & $\ln C=0.8767$ \\
Logarithmic & $R^{2}$ & 0.9259 \\
\hline
\end{tabular}

The main effect plots (Figure 3a) indicated that while increasing the AAc content up to a certain level decreased the drug load of samples, further uses of AAc in the formulation of hydrogels increased their drug absorption considerably. The reverse trend was also observed for silver nitrate and microwave exposure time, in which increasing these two parameters up to a certain level improved their drug loading efficiencies; however, additional contents of silver ions or extra duration of microwave irradiation decreased their drug absorption efficiencies. The contour plot of cephalexin absorption changes by AAc and silver nitrate (at a fixed middle level of microwave time was also shown in Figure 3b. According to Figure 3b, simultaneous increase or decrease of these two parameters could enhance the drug absorption efficiency of hydrogels.

The increase of drug load by AAc concentrations was an unusual observation, since at high monomer concentration the excessive crosslinking density would decrease the swelling ratio and absorption capacities of the obtained polymer. At high silver ion concentrations, a higher amount of carboxyl groups will be neutralized with silver ions. Thus, the polymerization rate will be lower than that of AAc polymerization due to the repulsion forces of negatively charged carboxylate groups during the propagation step of polymerization. As a result, at low silver concentrations, the polymerization rate will be higher and the hydrogel matrics will be more compact than that at high silver ion concentrations. Previous researches have shown that drug diffusion into hydrogels with high cross-linking densities matrices was difficult because the hydrogels with more cross-links were more compacted and possessed very small less penetrable pores [15]. Increasing the silver ions also ionized the background polymers, upturned their electrostatic repulsions, weakened their matrices, and made them more penetrable against drugs [16]. The weak cross-linked AAc-based hydrogels could be obtained at less microwave irradiation time. 

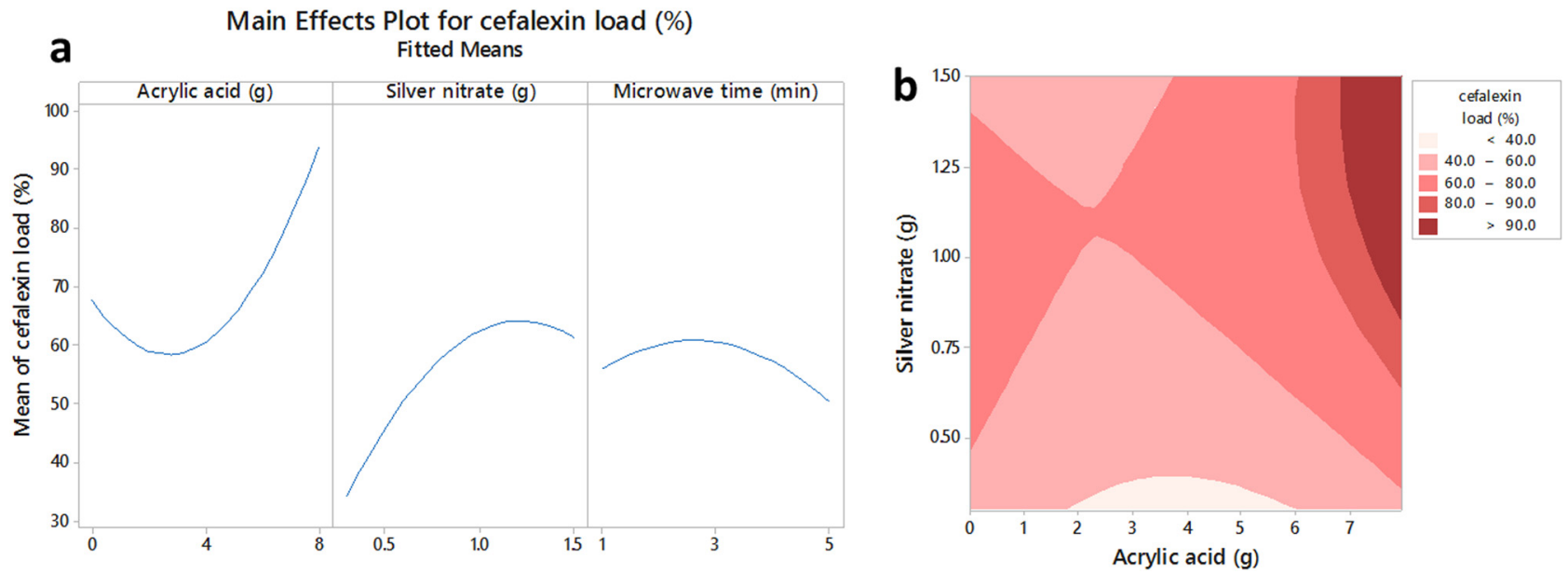

Figure 3: The main effects plot (a) and the contour plot (b) of acrylate acid, silver nitrate and microwave time on cefalexin load of sodium alginate/acrylic acid composite hydrogels conjugated to silver nanoparticles.

Thus, the pore size in the hydrogel matrix would be increased, causing a considerable increase in the efficiency of hydrogel absorption.

\subsection{Antibacterial activity}

All synthesized hydrogels showed antibacterial activity against $S$. aureus, with growth-inhibitory zone, ranged from 10 to $21 \mathrm{~mm}$. The ANOVA results of antibacterial activity designated that the linear effects of AAc and silver nitrate and the quadratic effect of AAc were just the significant terms, on changes of this characteristic in $95 \%$ confidence interval ( $p$-value $<0.05)$. The quadratic effect of silver nitrate content and also its interaction with microwave time were also significant in the $90 \%$ confidence interval $(0.1>p$-value $>0.05)$. The final reduced model after removing the insignificant terms $(p$-value $>0.1)$ was shown as Eq. 7 to predict the variation of antibacterial activity of hydrogels:

$$
\begin{aligned}
& \text { Growth inhibition zone }(\mathrm{mm}) \\
& =21.22+2.479 X_{1}-13.92 X_{2}-2.381 X_{3} \\
& -0.2239 X_{1}^{2}+7.90 X_{2}^{2}+2.35 X_{2} X_{3}
\end{aligned}
$$

According to obtained $R^{2}$ for Eq. $7\left(R^{2}=92.39 \%\right)$, it can be concluded that this model can predict more than $92 \%$ of antibacterial activities of hydrogels against S. aureus in terms of their growth-inhibited zone diameter.

The main effect plots (Figure 4a) for changes of this response by selected independent parameters point out a

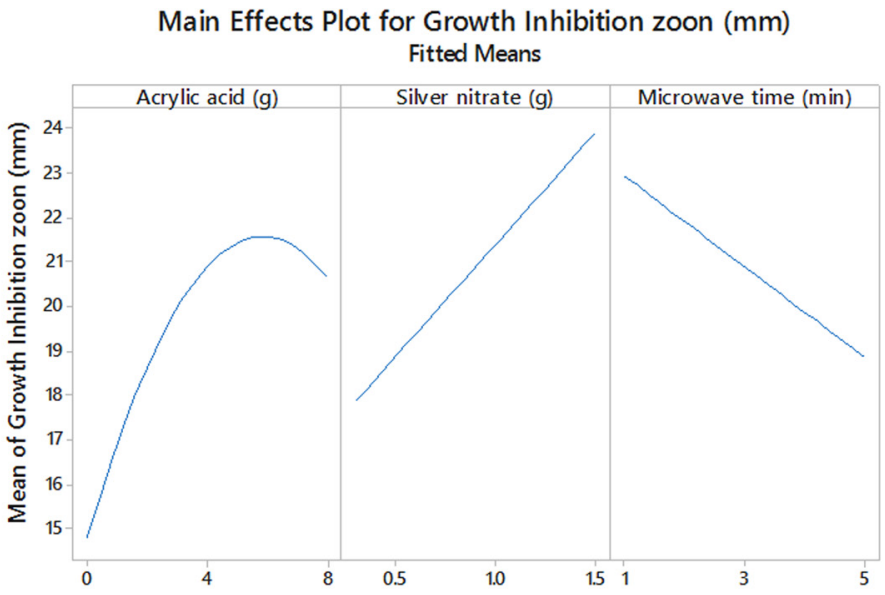

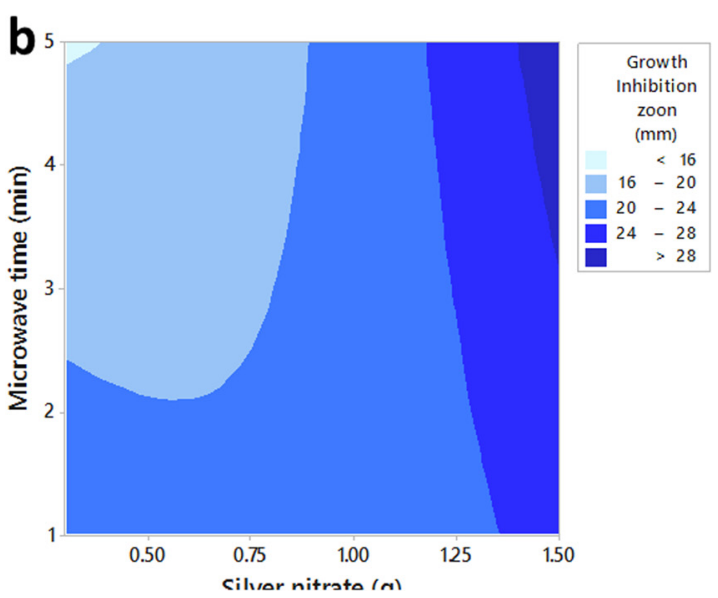

Figure 4: The main effects plot (a) and the contour plot (b) of acrylate acid, silver nitrate and microwave time on growth inhibition of sodium alginate/acrylic acid composite hydrogels conjugated to silver nanoparticles. 
that while increasing the AAc content up to a certain level increased the antibacterial activity of samples, further uses of AAc in the formulation of hydrogels affected reversely and decreased this ability of samples. Increasing the silver nitrate ions as well as decreasing the microwave time also strengthened the bactericidal activity of hydrogels. The contour plot of growth-inhibitory zoon of samples changed by silver nitrate content and microwave time (at a fixed middle level of AAc) was also shown in Figure 4b. As can be seen in Figure 4b, a simultaneous increase of both silver nitrate and microwave irradiation time could enhance the bactericidal activity of hydrogels.

According to the $F$-ratio of terms, the silver nitrate content was the most effective agent on the antibacterial activity of obtained hydrogels. This result was predictable due to the high antibacterial activity of silver. Some previous researches also reported an antibacterial activity for AAc and its di-block copolymers [18]. Thus, it seems that intensifying of hydrogels' antibacterial activities by increasing the AAc could be related to this antibacterial activity of AAc residues in hydrogel matrices. Increasing the irradiation time also decreased the antibacterial activity of hydrogels, due to increasing the cross-linking bonds between AAcs and decreasing the monomer residues. Considerable antibacterial activities of prepared samples at high silver nitrate contents and extended irradiation time could be related to the reduction of silver ions to silver nanoparticles by microwave emissions and their stabilization between the dense cross-linked matrices of hydrogels [19,20].

\subsection{Optimization and model confirmation}

The AAc and silver nitrate contents, as well as the microwave irradiation time, were numerically optimized to produce the hydrogels with the highest swelling ratio, gel fraction, cephalexin load, and S. aureus growth-inhibited zone, using multiple goal optimization processes. Thus, according to optimization analysis, using the highest contents of either AAc $(7.8 \mathrm{~g})$ or silver nitrate $(1.5 \mathrm{~g})$ at less microwave exposure time (1 $\mathrm{min})$ can give the hydrogels with the maximum highest swelling ratio (655\%), gel fraction $(>0.99)$, cephalexin load $(>0.99)$, and $S$. aureus growthinhibited zone $(24.97 \mathrm{~mm})$.

For confirming the presented models, three samples were prepared in obtained optimum conditions $(\mathrm{AAc}=7.8 \mathrm{~g}$, silver nitrate $=1.5 \mathrm{~g}$, and microwave exposure time $=1 \mathrm{~min}$ ) and were quantified. The swelling degree, gel fraction, cephalexin load, and antibacterial clear zone diameter of these samples were $650.5 \pm 14.5 \%, 0.98 \pm 0.07$,
$0.99 \pm 0.03 \%$, and $26 \pm 2 \mathrm{~mm}$, respectively. The insignificant differences between the experimental data and predicted ones by the model $(655 \%, 0.99,0.99 \%$, and $24.97 \mathrm{~mm}$, in turn), confirmed the suitability and correctness of models.

\subsection{Complementary characterizations of optimum sodium alginate/AAC composite hydrogels conjugated to silver nanoparticles}

\subsubsection{The absorption and release kinetics of cephalexin by optimum sodium alginate/AAC composite hydrogels conjugated to silver nanoparticles}

The absorption or release of bioactive compounds by or from most hydrogels is occurring mostly due to diffusion phenomena. However, the absorption/release profile for a swelled composite hydrogels is complex, depending on the relative diffusion rates of either water or bioactive compound from or toward hydrogel networks. If the diffusion into the hydrogel takes place at gentle rates as compared to the hydrogel chains' relaxation, the process will be controlled by diffusion. Otherwise, the process will be relaxation controlled. The irregular pattern between the mentioned states is known as non-Fickian diffusion.

To determine the cephalexin absorption kinetic model of the optimum composite hydrogel, they were immersed into an aqueous solution containing a certain amount of cephalexin $\left(0.25 \mathrm{~g} \cdot \mathrm{L}^{-1}\right)$, for $5 \mathrm{~h}$, and cephalexin content of aqueous media was measured at fixed time intervals. The results were correlated to common kinetics models, namely zero-order, first-order, second-order, Michaelis-Menten, and logarithmic models. The linearized model coefficients and coefficient of determination $\left(R^{2}\right)$ for each model were calculated. These results were shown in Table 3. Due to the highest obtained $R^{2}$ for zero-order kinetics, it can be concluded that the cephalexin absorption by synthesized optimum composite hydrogels obeyed the zero-order kinetic model. Thus, this model was chosen as the best model to describe the absorption behavior of optimum hydrogel for cephalexin at its studied concentrations. In zero-order kinetics, the absorption only depends on time and is constant at various concentrations of the active compounds [21]. The absorption occurs rapidly until the saturation concentration at the equilibrium state reaches. The electrostatic interactions occurred between the functional group (COO-) of composite hydrogel like $-\mathrm{COOH}$ and $-\mathrm{OH}$ groups, $\mathrm{Ag} \mathrm{NP}$, and cephalexin like $-\mathrm{OH},-\mathrm{NH}_{2},-\mathrm{S}-$, 
Table 4: The kinetics of cephalexin release from optimum composite hydrogels (the $R^{2}$ and main coefficients for correlated general release models)

\begin{tabular}{llll}
\hline & Common release models & & Cephalexin release \\
\hline Zero order & $C-C_{0}=-K t$ & Equation & $C=0.1009 t+0.0741$ \\
& Linear & $R^{2}$ & 0.9514 \\
First order & $\ln C-\ln C_{0}=K t$ & Equation & $\ln C=0.112 t-0.8406$ \\
& First order & $R^{2}$ & 0.7979 \\
Higuchi model & $C-C_{0}=K t^{0.5}$ & Equation & $C=0.3522 t^{(0.5)}-0.173$ \\
Hixson Crowell & & $R^{2}$ & 0.9977 \\
& $C^{1 / 3}-C_{0}^{1 / 3}=K t$ & $C^{(1 / 3)}=0.0533 t+0.5849$ & 0.735 \\
Korsmeyer Peppas model (power law model) & $\ln C-\ln C_{0}=K \ln t$ & $R^{2}$ & $\ln C=0.8183 \ln t+1.8078$ \\
& Logarithmic & Equation & 0.9872 \\
\hline
\end{tabular}

and $=0$. Particularly, the hydrogen bonding between the cephalexin and hydrogel sub-unites plays a vital role in their absorption efficiencies. The possible presence of cations residues like $\mathrm{Ag}^{+}$and $\mathrm{Ca}^{2+}$ in hydrogel structure also can increase the affinity of negative surface parts of cephalexin into the hydrogel matrices [22].

The release kinetics of cephalexin from optimum hydrogels (into deionized water, $\mathrm{pH}=7.0 \pm 0.1$ ) was also evaluated after completely saturation and removal of accumulated surface molecules of cephalexin. The cephalexin contents of deionized water were measured at certain time intervals. Similar to the cephalexin absorption survey, the results were correlated to common kinetics models, and the obtained linearized model coefficients and coefficient of determination $\left(R^{2}\right)$ for each model were summarized in Table 4. From Table 4 and obtained $R^{2}$ values for each model, due to the greatest $R^{2}$ for the Higuchi model, it can be concluded that the release of cephalexin from the synthesized hydrogels follows this model. Based on the Higuchi model, the release is considered as a diffusion process based on Fick's law. The diffusion occurs at a microscopic or molecular scale through the hydrogel networks, and the diffusion rate decreases as the release process continues because the drug should pass an elongated path to reach the media and be released. The Higuchi model is valid for most of the water-soluble or water-insoluble drugs that are released from semisolid matrices like hydrogels [23].

\subsubsection{FT-IR and UV-Vis absorbance spectrum of optimum hydrogels}

The FT-IR spectra of the optimum sodium alginate/AAc composite hydrogels conjugated to silver nanoparticles were shown in Figure 5a. The absorption spectrum from
3,950 to $3,500 \mathrm{~cm}^{-1}$ was pointed out to the stretching sodium alginate $\mathrm{NH}_{2}$. The peak at $3,431 \mathrm{~cm}^{-1}$ was related to the hydroxyl group. The broad peak from 3,350 to $2,500 \mathrm{~cm}^{-1}$ indicated the presence of $\mathrm{OH}$ scratching of AAc. The peaks at 1,634 and $1,433 \mathrm{~cm}^{-1}$ were appeared, respectively, due to symmetric stretching vibration of the carboxylate group. The low peak at $1,500 \mathrm{~cm}^{-1}$ was assigned to stretching $\mathrm{C}-\mathrm{O}$. The peaks from 1,650 to $1,750 \mathrm{~cm}^{-1}$ were corresponded to stretching vibration of the carbonyl group of carboxylic acid, confirming the

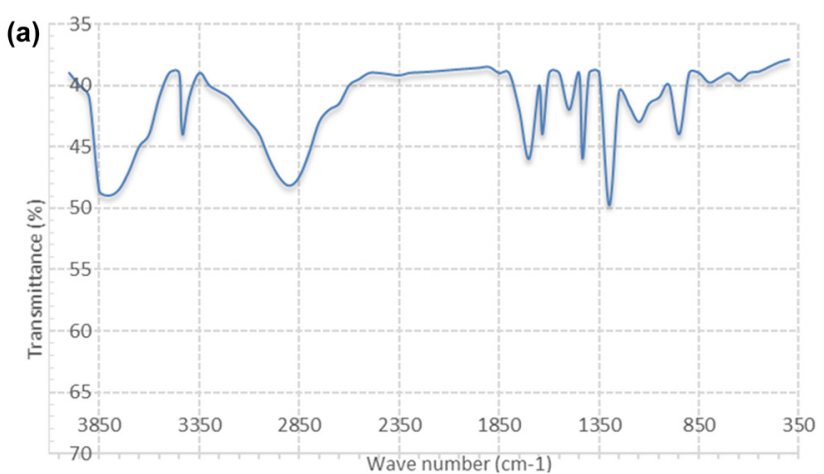

(b)

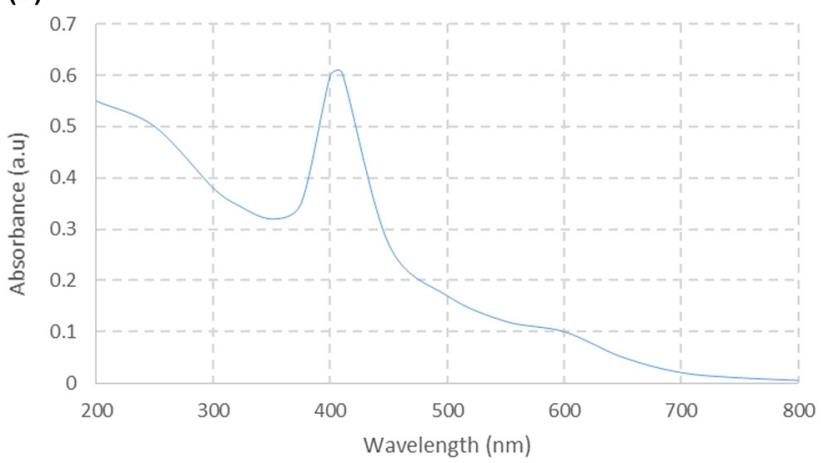

Figure 5: FT-IR (a) and UV-Vis absorbance (b) spectra of the optimum sodium alginate/AAc composite hydrogels conjugated to silver nanoparticles. 
presence of poly(AAc) in product. The broadband from 1,300 to $1,000 \mathrm{~cm}^{-1}$ was referred to the stretching vibration of $\mathrm{C}-\mathrm{O}$ bonds of alginate. The peak at $950 \mathrm{~cm}^{-1}$ was related to $\mathrm{O}-\mathrm{H}$ bonding of polysaccharide monomers in hydrogel. Moreover, the small band at $819 \mathrm{~cm}$ is attributed to the $\mathrm{Na}-\mathrm{O}$ bond in sodium alginate $[24,25]$.

Figure $5 \mathrm{~b}$ shows the UV-Vis absorbance spectra of optimum composite hydrogels conjugated to silver nanoparticles. The observed peak at about $400 \mathrm{~nm}$ was related to the surface plasmon resonance of silver nanoparticles.
Thus, the formation of silver nanoparticles from silver ions inside the hydrogel matrices was confirmed [20].

\subsubsection{SEM images of optimum hydrogels}

The surface morphology of optimum hydrogel was visualized by SEM images, which were taken in different scales from $100 \mu \mathrm{m}$ to $500 \mathrm{~nm}$ (Figure 6). The hydrogels' SEM images showed the relatively wide-sized distribution of
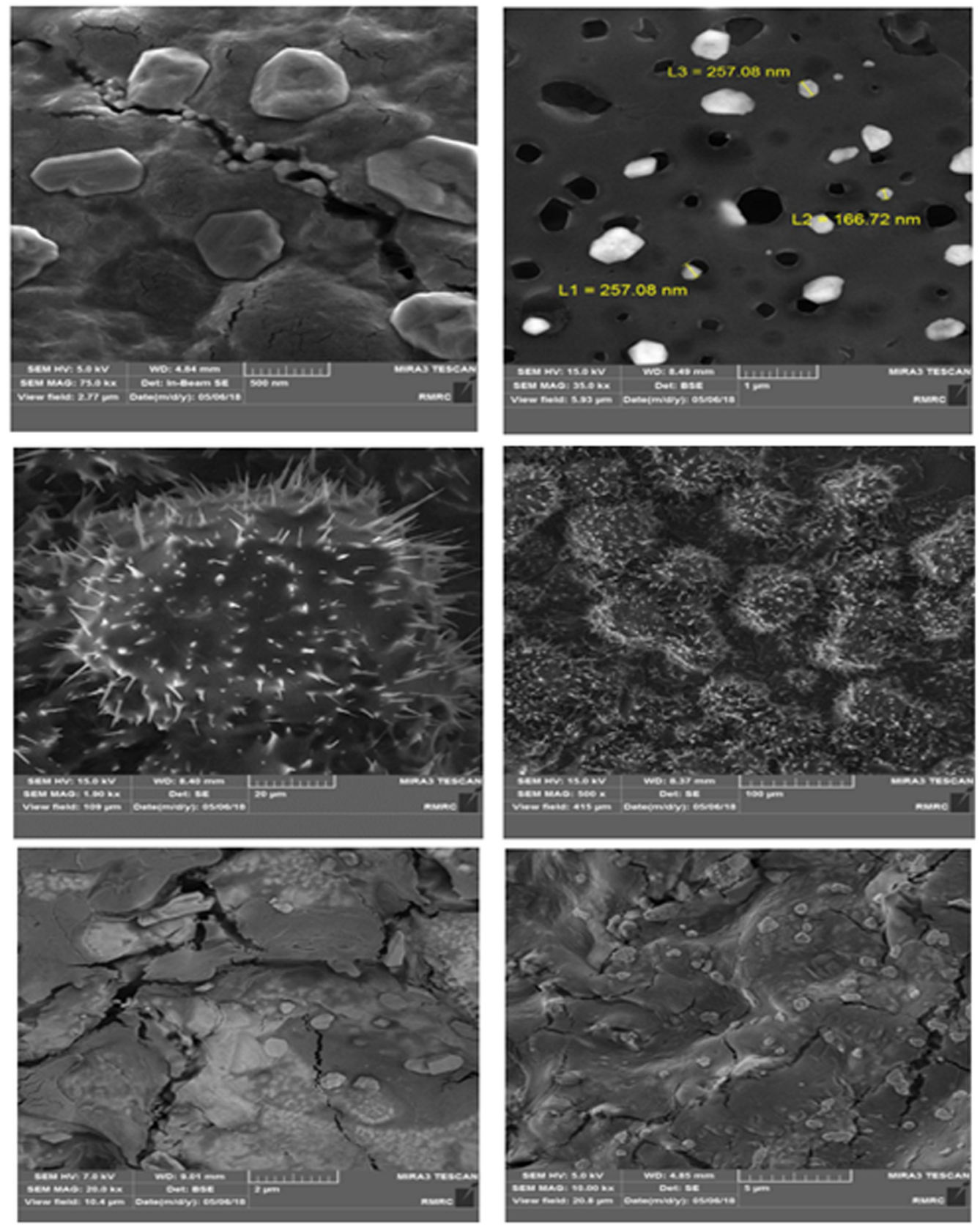

Figure 6: FE-SEM images of the optimum sodium alginate/AAc composite hydrogels conjugated to silver nanoparticles. 
produced silver nanoparticles on the surface of the hydrogel. The presence of fine pores on hydrogels' surface, which can be seen in TEM images, made them efficient candidates for taking the various bioactive compounds. The energy-dispersive $\mathrm{X}$-ray spectrum of samples also confirmed the presence of $\mathrm{Ag}, \mathrm{Ca}, \mathrm{Cl}, \mathrm{Na}, \mathrm{N}, \mathrm{O}$, and $\mathrm{C}$ on the surface of prepared hydrogels.

\section{Conclusion}

The physical microwave radiation and ion cross-linking process were simultaneously applied to synthesis the sodium alginate/AAc composite hydrogels and reduce the incorporated silver ions into stable silver nanoparticles, which were conjugated to hydrogel matrices. Response surface methodology was used for experimental design, statistical analysis, developing the models, and optimizing the fabrication parameters of gained hydrogels. The hydrogels with the highest swelling ratio, gel fraction, cephalexin absorption, and antibacterial activities were considered as the most desired product, and the fabrication parameters were optimized to achieve the best sodium alginate/AAc composite hydrogels. The absorption and release kinetics of cephalexin from the optimum product were also investigated. It resulted that while the drug absorption obeys the zero-order kinetic model, the release process could be explained by Higuchi kinetic model. Due to the high antibacterial activity of prepared composite hydrogels, which could be resulted from the synergistic effect of silver ions, AAc, and cephalexin, they can be successfully used in various medical and pharmaceutical applications such as wound healing lotions or wound dressing formulations.

Funding information: Authors state no funding involved.

Author contributions: Parvaneh Mohamadinia: data curation, formal analysis, funding acquisition, investigation, methodology, validation, and writing - original draft; Navideh Anarjan: conceptualization, data curation, formal analysis, investigation, methodology, project administration, software, supervision, validation, visualization, and writing - review and editing; and Hoda Jafarizadeh-Malmiri: validation, visualization, and writing - review and editing.

Conflict of interest: Authors state no conflict of interest.

Data availability statement: The data that support the findings of this study are available from the corresponding author on reasonable request.

\section{References}

[1] Parhi R. Cross-linked hydrogel for pharmaceutical applications: a review. Adv Pharm Bull. 2017;7(4):515-30. doi: 10.15171/apb.2017.064.

[2] Mori Z, Anarjan N. Preparation and characterization of nanoemulsion based $\beta$-carotene hydrogels. J Food Sci Tech. 2018;55:5014-24. doi: 10.1007/s13197-018-3440-3.

[3] Thakur S, Sharma B, Verma A, Chaudhary J, Tamulevicius S, Thakur VK. Recent progress in sodium alginate based sustainable hydrogels for environmental applications. J Clean Prod. 2018;198:143-59. doi: 10.1016/ j.jclepro.2018.06.259.

[4] Gaharwar A, Peppas N, Khademhosseini A. Nanocomposite hydrogels for biomedical applications. Biotechnol Bioeng. 2014;111:441-53. doi: 10.1002/bit.25160.

[5] Dannert C, Stokke BT, Dias RS. Nanoparticle-hydrogel composites: from molecular interactions to macroscopic behavior. Polymers. 2019;11(2):275. doi: 10.3390/polym11020275.

[6] Serrano-Aroca Á, Deb S. Acrylic-based hydrogels as advanced biomaterials. In: Serrano-Aroca Á, Deb S, editors. Acrylate polymers for advanced applications. London: IntechOpen; 2020. p. 1-23

[7] Tang S, Zheng J. Antibacterial activity of silver nanoparticles: structural effects. Adv Healthc Mater. 2018;7(13):e1701503. doi: 10.1002/adhm.201701503.

[8] Patra JK, Baek K-H. Antibacterial activity and synergistic antibacterial potential of biosynthesized silver nanoparticles against foodborne pathogenic bacteria along with its anticandidal and antioxidant effects. Front Microbiol. 2017;8:167. doi: $10.3389 /$ fmicb.2017.00167.

[9] Anarjan N, Nehdi IA, Tan CP. Influence of astaxanthin, emulsifier and organic phase concentration on physicochemical properties of astaxanthin nanodispersions. Chem Cent J. 2013;7(1):127. doi: 10.1186/1752-153X-7-127.

[10] Kondaveeti S, Bueno PVDA, Carmona-Ribeiro AM, Esposito F, Lincopan N, Sierakowski MR, et al. Microbicidal gentamicinalginate hydrogels. Carbohydr Polym. 2018;186:159-67. doi: 10.1016/j.carbpol.2018.01.044.

[11] Wang T, Zhang F, Zhao R, Wang C, Hu K, Sun Y, et al. Polyvinyl alcohol/sodium alginate hydrogels incorporated with silver nanoclusters via green tea extract for antibacterial applications. Des Monomers Polym. 2020;23(1):118-33. doi: 10.1080/ 15685551.2020.1804183.

[12] Ganji F, Vasheghani Farahani S, Vasheghani-Farahani E. Theoretical description of hydrogel swelling: a review. Iran Polym J. 2010;19:375-98.

[13] Makhado E, Pandey S, Ramontja J. Microwave assisted synthesis of xanthan gum-cl-poly (acrylic acid) based-reduced graphene oxide hydrogel composite for adsorption of methylene blue and methyl violet from aqueous solution. Int J Biol Macromol. 2018;119:255-69. doi: 10.1016/ j.ijbiomac.2018.07.104.

[14] Kretschmann O, Schmitz S, Ritter H. Microwave-assisted synthesis of associative hydrogels. Macromol Rapid Commun. 2017;28:1265-9. doi: 10.1002/marc.200700117.

[15] Kowalski G, Kijowska K, Witczak M, Kuterasiński t, tukasiewicz $M$. Synthesis and effect of structure on swelling properties of hydrogels based on high methylated pectin and 
acrylic polymers. Polymers. 2019;11(1):114. doi: 10.3390/ polym11010114.

[16] Quintanilla de Stéfano JC, Abundis-Correa V, HerreraFlores SD, Alvarez AJ. pH-sensitive starch-based hydrogels: synthesis and effect of molecular components on drug release behavior. Polymers. 2020;12(9):1974. doi: 10.3390/ polym12091974.

[17] Pourjavadi A, Barzegar S, Mahdavinia G. MBA-crosslinked NaAlg/CMC as a smart full-polysaccharide superabsorbent hydrogel. Carbohydr Polym. 2006;66:386-95. doi: 10.1016/ j.carbpol.2006.03.013.

[18] Gratzl G, Paulik C, Hild S, Guggenbichler JP, Lackner M. Antimicrobial activity of poly (acrylic acid) block copolymers. Mater Sci Eng C. 2014;38:94-100. doi: 10.1016/ j.msec.2014.01.050.

[19] Xia B, Cui Q, He F, Li L. Preparation of hybrid hydrogel containing ag nanoparticles by a green in situ reduction method. Langmuir. 2012;28(30):11188-94. doi: 10.1021/la302011x.

[20] Singh J, Dhaliwal AS. Water retention and controlled release of $\mathrm{KCl}$ by using microwave-assisted green synthesis of xanthan gum-cl-poly (acrylic acid)/AgNPs hydrogel nanocomposite. Polym. 2020;77(9):4867-93. doi: 10.1007/s00289-01902990-x.
[21] Bhasarkar J, Bal D. Kinetic investigation of a controlled drug delivery system based on alginate scaffold with embedded voids. J Appl Biomater Funct Mater. 2019;17(2):2280800018817462. doi: $10.1177 / 2280800018817462$.

[22] Verma A, Thakur S, Mamba G, Prateek, Gupta RK, Thakur P, et al. Graphite modified sodium alginate hydrogel composite for efficient removal of malachite green dye. Int J Biol Macromol. 2020;148:1130-9. doi: 10.1016/ j.ijbiomac.2020.01.142.

[23] Peppas NA, Narasimhan B. Mathematical models in drug delivery: how modeling has shaped the way we design new drug delivery systems. J Control Release. 2014;190:75-81. doi: 10.1016/j.jconrel.2014.06.041.

[24] Bekin S, Sarmad S, Gürkan K, Keçeli G, Gürdağ G. Synthesis, characterization and bending behavior of electroresponsive sodium alginate/poly(acrylic acid) interpenetrating network films under an electric field stimulus. Sens Actuators B Chem. 2014;202:878-92. doi: 10.1016/ j.snb.2014.06.051.

[25] Bekin S, Sarmad S, Gürkan K, Keçeli G, Gürdağ G. Dielectric, thermal, and swelling properties of calcium ion-crosslinked sodium alginate film. Polym Eng Sci. 2014;54:1372-82. doi: $10.1002 /$ pen.23678. 